\title{
Article \\ Finitely Supported Binary Relations between Infinite Atomic Sets
}

\author{
Andrei Alexandru ${ }^{1}$ and Gabriel Ciobanu ${ }^{2, *(D)}$ \\ 1 Institute of Computer Science, Romanian Academy, 700505 Iaşi, Romania; \\ andrei.alexandru@iit.academiaromana-is.ro \\ 2 Faculty of Computer Science, Alexandru Ioan Cuza University, 700506 Iaşi, Romania \\ * Correspondence: gabriel@info.uaic.ro
}

Citation: Alexandru, A.; Ciobanu, G. Finitely Supported Binary Relations between Infinite Atomic Sets. Symmetry 2021, 13, 2028. https:// doi.org/10.3390/sym13112028

Academic Editor: José Carlos R. Alcantud

Received: 8 October 2021

Accepted: 21 October 2021

Published: 27 October 2021

Publisher's Note: MDPI stays neutral with regard to jurisdictional claims in published maps and institutional affiliations.

Copyright: (c) 2021 by the authors. Licensee MDPI, Basel, Switzerland. This article is an open access article distributed under the terms and conditions of the Creative Commons Attribution (CC BY) license (https:// creativecommons.org/licenses/by/ $4.0 /)$.

\begin{abstract}
In the framework of finitely supported atomic sets, by using the notion of atomic cardinality and the $T$-finite support principle (a closure property for supports in some higher-order constructions), we present some finiteness properties of the finitely supported binary relations between infinite atomic sets. Of particular interest are finitely supported Dedekind-finite sets because they do not contain finitely supported, countably infinite subsets. We prove that the infinite sets $\wp_{f_{\mathcal{S}}}\left(A^{k} \times A^{l}\right), \wp_{f_{s}}\left(A^{k} \times \wp_{m}(A)\right), \wp_{f_{s}}\left(\wp_{n}(A) \times A^{k}\right)$ and $\wp_{f_{\mathcal{S}}}\left(\wp_{n}(A) \times \wp_{m}(A)\right)$ do not contain uniformly supported infinite subsets. Moreover, the functions space $Z^{A^{m}}$ does not contain a uniformly supported infinite subset whenever $Z$ does not contain a uniformly supported infinite subset. All these sets are Dedekind-finite in the framework of finitely supported structures.
\end{abstract}

Keywords: finitely supported structures; atomic sets; relations; cardinality; finiteness properties

MSC: 03E25; 03E30; 03B70

\section{Introduction}

\subsection{Motivation}

The framework of this article is given by the finitely supported structures. These structures are related to the permutation models of Zermelo-Fraenkel set theory with atoms [1,2]. Permutation models were introduced in 1930s by Fraenkel, Mostowski and Lindenbaum $[3,4]$ for proving the independence of the axiom of choice from the other axioms of Zermelo-Fraenkel set theory with atoms (ZFA). More recently, finitely supported sets have been developed in Zermelo-Fraenkel set theory (ZF), by equipping ZF sets with actions of a group of permutations of some basic elements called atoms. They were used to investigate the variables binding, renaming and freshness in the theory of programming [5-7]. Inductively defined finitely supported sets that involve the name-abstraction together with disjoint unions and Cartesian products can encode a formal syntax modulo renaming of bound variables. In this manner, the theory of algebraic data types can be extended to include signatures involving binding operators. In particular, the notions of structural recursion and structural induction can be consistently modelled in this new framework [7].

Finitely supported sets that are equipped with finitely supported internal operations represent finitely supported structures described in $[5,6]$. These finitely supported structures are of interest for computer scientists because they present a way of computing very large (possibly infinite) structures having a certain degree of symmetry; this means that they allow a computational study of possibly infinite sets containing enough symmetries to be concisely handled. Actually, this theory allows us to treat as equivalent the objects those that have a certain degree of similarity and to focus only on those objects that are 'really different'. 
Regarding the notion of finite support, in a lambda-calculus interpretation the finite support of a lambda-term is represented precisely by the set of all free variables of the term; these variables are those who really matter in order to characterize the term, while the other (bound) variables can simply be renamed. Therefore, by renaming its bound variables, we can construct an infinite family of terms starting from an original one, but in order to handle this infinite family it is enough to work with the finite set of free variables (i.e., the support) of the original term. By using the finitely supported structures, we are able to deal with infinite sets and to provide precise descriptions of very large structures by using symmetry orbits [6,7].

In this article we extend the approach presented in [8] where various forms of infinity for finitely supported sets were introduced and compared. More exactly, we present some (Dedekind-) finiteness properties of finitely supported (binary) relations between infinite atomic sets in the framework of finitely supported structures. If $S$ is a finite set of atoms, there exists only a finite number of $S$-supported binary relations between some large infinite atomic sets.

\subsection{Methodology}

An invariant set is defined as a usual ZF set endowed with a group action of the group of all (finitary) permutations over a certain fixed infinite ZF set $A$ of basic elements whose internal structure is ignored (called atoms) satisfying a finite support requirement. This related finite support requirement says that any element in an invariant set must be finitely supported, i.e., for any such element there should exist a finite set of atoms such that any permutation of atoms fixing pointwise this finite set of atoms also leaves the element invariant under the related group action. An 'invariant set' corresponds to a 'nominal set' [7] if the set $A$ of atoms is countable. A finitely supported set is defined as a finitely supported element in the powerset of an invariant set. A finitely supported structure is defined as a finitely supported set equipped with a finitely supported internal operation or relation (which should be finitely supported as a subset of a Cartesian product of finitely supported sets). Formal definitions are presented in Section 2.

The framework of finitely supported structures contains both the family of 'nonatomic' (i.e., classical) ZF structures (hierarchically constructed over $\varnothing$ ) which are proved to be trivially invariant structures (i.e., all their elements are empty supported) and the family of 'atomic' structures (i.e., structures that are hierarchically constructed by involving $\varnothing$ and atoms). Our goal is to investigate whether a non-atomic ZF result preserves its validity when it is reformulated by replacing 'non-atomic ZF structure' with 'atomic and finitely supported structure'. As proved in [6], such a translation is not obvious and some results (like various relationship results between choice principles or Stone duality) cannot be translated from ZF into the new atomic framework. The proof of each new result should be internally consistent in the framework of finitely supported structures and not retrieved from ZF; this means that it should involve only finitely supported constructions, even in the intermediate steps of a proof. The meta-theoretical techniques for the translation of a result from non-atomic structures to atomic structures are based on a closure property for finite supports in a hierarchical construction, property called ' $T$-finite support principle'. More details are presented in Section 2.

A related meta-theoretical approach was used in [8] to prove some properties of finitely supported cardinalities used in this paper to present counting properties for finitely supported binary relations between infinite atomic sets. For proving our results, we also use a freshness property saying that for any element $x$ in a finitely supported set it is possible to find a 'fresh' atom $a$, i.e., an atom $a$ which is outside the support of $x$.

\subsection{Literature Review}

Fraenkel introduced the permutation method and model in 1922 to prove the independence of the axiom of choice from the other axioms of a set theory with atoms [3]; Fraenkel's model was refined and extended by Lindenbaum and Mostowski in [4]. Around 1940 
Godel proved that the axiom of choice is consistent with the axioms of von-NeumannBernays-Godel set theory. Cohen proved in 1963 the independence of the axiom of choice from the standard axioms of ZF set theory [2]. We continued this research line in [5,6]; by using atomic permutation actions we introduced finitely supported sets and structures which are consistent according to ZF axioms (it is not necessary to weaken the axioms of extensionality in order to allow the existence of atoms). We extensively studied several choice principles and got results related to various forms of choice, infinity, fixed points, approximation and stability for finitely supported structures. We also investigated the consistency of the translation of the non-atomic results into the framework of finitely supported sets, and present specific properties of atomic sets.

Finitely supported sets are also connected to the recent development of FraenkelMostowski (FM) axiomatic set theory [9] which represents an axiomatization of Fraenkel basic model for Zermelo-Fraenkel set theory with atoms (model N1 from [10]), and to the theory of admissible sets of Barwise (particularly to the theory of hereditary finite sets [11]). Nominal sets, described in [7], represent a ZF alternative to the non-standard FM set theory, because nominal sets are defined by involving group actions over standard ZF sets without being necessary to modify the ZF axioms of extensionality or foundation. Nominal sets also serve as a good framework to model the syntax of formal systems involving variablebinding operations as well as a framework for database theory because atoms can be used as an abstraction for data values appearing in a relational database. Atoms can also be used to model sources of infinite data in some applications such as software verification, where an atom can represent a pointer or the contents of an array cell. Atoms have the same properties as names; the precise nature of names is unimportant because we are focused only on their distinctness and on their ability to identify. Nominal sets have also been used in game theory [12], in topology [13], in proof theory [14] where is developed a proof assistant based on nominal sets, and in process calculi [15] where the $\pi$-calculus was formalized in Isabelle by using the nominal datatypes. Generalizations of nominal sets are involved in the study of automata, Turing machines and programming languages over infinite alphabets; for this, a more relaxed notion of finiteness named 'orbit finiteness' was defined as 'having a finite number of orbits (equivalence classes) under a certain group action' [16].

The invariant sets are defined over possibly non-countable sets of atoms; they are also motivated by Tarski's approach regarding logicality (a logical notion is defined by Tarski as one that is invariant under the one-to-one and onto transformations of the universe of discourse [17]). The concept of 'invariant structure' was described and investigated in [5]. A more general concept of 'finitely supported structure' for arbitrary algebraic structures (that are not necessarily partially ordered sets) was described and investigated in [6]. New results (such as generalized Tarski fixed point theorems) were also proved for invariant partially ordered sets.

Other generalizations of finitely supported sets include Fraenkel-Mostowski generalized set theory (FMG) introduced in [9] by generalizing both the size of atoms and the size of support from the FM set theory. More exactly, FMG is a generalization of the FM sets framework obtained by replacing 'finite support' with 'well-orderable (but at least countable) support' and by considering an uncountable set of atoms. The differences between this approach and the framework of invariant sets are explained in [6]. Private names in non-commutative logic [18] are modelled by the de Morgan dual pair of quantifiers generalizing the nominal quantifier that encode 'all, but finitely many' [7]. These quantifiers are polarized in the sense that one of them distributes over positive operators, while the other one distributes over negative operators. This enables private names to be modelled in processes embedded as predicates in an expressive but decidable proof system named MAV1. 


\subsection{Our Contribution}

By using several results obtained by the authors in the theory of finitely supported structures [6,8], here there are described some apparently large sets (such as finite powersets, functions spaces and families of relations between infinite atomic sets) satisfying some (Dedekind-) finiteness properties. In ZF, a set $X$ is Dedekind-finite if and only if every injective self-function on $X$ is also surjective. Equivalently, a set is Dedekind-finite if and only if it does not contain an infinite countable subset. These results are preserved in the framework of finitely supported sets and structures. More exactly, a finitely supported set $(X, \diamond)$ is Dedekind-finite if and only if every finitely supported injective self-function on $X$ is also surjective. As a characterization, a finitely supported set is Dedekind-finite if and only if it does not contain a finitely supported countable subset. We proved in [8] that a countable finitely supported set is necessarily uniformly supported (i.e., every element of the set is supported by the same finite set of atoms); thus, a finitely supported set that does not contain a uniformly supported infinite subset is Dedekind-finite. In this paper we prove stronger results than in [8], results claiming that $X^{A^{m}}$ is Dedekind-finite for each $m \in \mathbb{N}$ whenever $X$ is a finitely supported set that does not contain a uniformly supported infinite subset; in particular, $X$ could be $\wp_{f s}(A), A_{f_{s}}^{A}$ or $\wp_{f s}(A)_{f_{s}}^{A}$. Moreover, the sets of all finitely supported binary relations between $A^{k}, \wp_{m}(A), \wp_{n}(A)$ and $A^{l}$, with $k, m, n, l \in \mathbb{N}$ are also Dedekind-finite.

\section{Preliminary Results}

We mention some preliminary results that are involved in the proofs of the original results presented in this article; several results belong to the authors of this paper.

We consider a standard Zermelo-Fraenkel infinite set $A$ (called the set of atoms), and ignore the internal structure of its elements. A transposition is defined as a function $(x y): A \rightarrow A$ having the properties $(x y)(y)=x,(x y)(x)=y,(x y)(z)=z$ for $z \neq x, y$. A permutation of $A$ is a bijection of $A$ obtained by composing finitely many transpositions, i.e., a bijection of $A$ leaving unchanged all but finitely many elements of $A$. By $P_{A}$ is denoted the set of all permutations of $A$.

Definition 1. Let $X$ be a set in the Zermelo-Fraenkel set theory (ZF).

1. $A P_{A}$-action on $X$ represents a group action of $P_{A}$ on $X$, i.e., a function $\diamond: P_{A} \times X \rightarrow X$ that satisfies $I d_{A} \diamond x=x$ and $\pi \diamond\left(\pi^{\prime} \diamond x\right)=\left(\pi \circ \pi^{\prime}\right) \diamond x$ for all $\pi, \pi^{\prime} \in P_{A}$ and $x \in X$.

2. $A P_{A}$-set is a pair $(X, \diamond)$, with $X$ a $Z F$ set and $\diamond: P_{A} \times X \rightarrow X$ a $P_{A}$-action on $X$.

3. Considering a $P_{A^{-}}$set $(X, \diamond)$, we say that $T \subset A$ supports $x$ (or that $x$ is $T$-supported) if $\pi \diamond x=x$ for each $\pi \in \operatorname{Inv}(T)$, where $\operatorname{Inv}(T)=\{\pi \mid \pi(a)=a$ for all $a \in T\}$. An element supported by a finite subset of atoms is called finitely supported.

4. Considering a $P_{A}$-set $(X, \diamond)$, we say that $X$ is an invariant set if for each $x \in X$ there exists a finite set $T_{x} \subset A$ supporting $x$.

5. Considering a $P_{A}$-set $(X, \diamond)$ and $x \in X$, we know from [5] that there is a least finite set $\operatorname{supp}(x)$ supporting $x$ whenever there is a finite set supporting $x$. This finite $\operatorname{set} \operatorname{supp}(x)$ supporting $x$ is the intersection of all sets supporting $x$, and it is called the support of $x$.

6. An empty supported element is equivariant. Thus, $z \in X$ is equivariant if and only if $\pi \diamond z=z$ for all $\pi \in P_{A}$.

Fraenkel-Mostowski sets were introduced in [4] as sets with atoms that are hereditary finitely supported (i.e., they are finitely supported, all their elements are finitely supported, and so on). They were firstly considered in [3] in order to study the independence of the axiom of choice in ZFA. ZFA theory is a refinement of ZF theory: in ZFA there does not exist only a basic element $\varnothing$, but infinitely many basic elements (having no internal structure) named atoms which can be compared only for equality. Moreover, the axiom of extensionality from ZF is modified in ZFA to allow atoms; more precisely, the ZFA axiom of extensionality states that 'any sets that are not atoms are equal if and only if they have the same elements'. 
In defining our finitely supported sets, we are able to work directly over ZermeloFraenkel set theory (ZF), without being necessary to avoid or modify the axiom of extensionality. In this sense, we adapted the notion of Fraenkel-Mostowski set to our context, and defined the invariant sets as sets equipped with a group action of the group of all permutations of $A$ having an additional finite support property for all its elements. An invariant set corresponds to a nominal set of [7] whenever $A$ is countable. By analogy, an invariant set corresponds to an empty supported (equivariant) set in the class of all Fraenkel-Mostowski sets defined as a von-Neumann cumulative hierarchy over $\varnothing$ and $A$.

Let $(X, \diamond)$ and $(Y, \triangleright)$ be $P_{A}$-sets. Based on [5], the set $A$ of atoms is an invariant set where the $P_{A}$-action $\diamond: P_{A} \times A \rightarrow A$ is defined by $\pi \diamond a:=\pi(a)$ for all $\pi \in P_{A}$ and $a \in A$. Whenever $\pi \in P_{A}$ and $x \in X$ is finitely supported, $\pi \diamond x$ is also finitely supported, also having the property $\operatorname{supp}(\pi \diamond x)=\{\pi(y) \mid y \in \operatorname{supp}(x)\}:=\pi(\operatorname{supp}(x))$.

The Cartesian product $X \times Y$ is a $P_{A}$-set with the $P_{A}$-action $\otimes$ defined by $\pi \otimes(x, y)=$ $(\pi \diamond x, \pi \triangleright y)$ for all $\pi \in P_{A}$ and $x \in X, y \in Y$; for invariant sets $(X, \diamond)$ and $(Y, \triangleright),(X \times Y, \otimes)$ is also an invariant set. The powerset $\wp(X)=\{Z \mid Z \subseteq X\}$ is a $P_{A}$-set with the $P_{A}$-action $\star: P_{A} \times \wp(X) \rightarrow \wp(X)$ defined by $\pi \star Z:=\{\pi \diamond z \mid z \in Z\}$ for all $\pi \in P_{A}$ and $Z \subseteq X$. For an invariant set $(X, \diamond), \wp_{f_{s}}(X)$ denotes the set formed from those subsets of $X$ that are finitely supported according to Definition $1(3)$ in $\wp(X)$ with respect to the action $\star$; $\left(\wp_{f_{s}}(X),\left.\star\right|_{\wp_{f s}(X)}\right)$ is also an invariant set, with $\left.\star\right|_{\wp_{f s}}(X)$ representing the action $\star$ restricted to $\wp_{f_{s}}(X)$. Non-atomic sets are trivially invariant; they are equipped with the trivial $P_{A}$-action $(\pi, x) \mapsto x$. The disjoint union of $X$ and $Y$ is given by $X+Y=\{(0, x) \mid x \in$ $X\} \cup\{(1, y) \mid y \in Y\} ; X+Y$ is a $P_{A}$-set with the $P_{A}$-action $\star$ defined by $\pi \star z=(0, \pi \diamond x)$ if $z=(0, x)$ and $\pi \star z=(1, \pi \triangleright y)$ if $z=(1, y)$. Thus, $(X+Y, \star)$ is an invariant set whenever $X$ and $Y$ are invariant sets.

\section{Definition 2.}

1. A subset $Z$ of an invariant set $(X, \diamond)$ is finitely supported if and only if $Z \in \wp_{f s}(X)$, namely if and only if $Z$ is finitely supported as an element of the $P_{A}$-set $(\wp(X), \star)$. In this case, we state that $(Z, \diamond)$ is a finitely supported set.

2. A subset $Z$ of an invariant set $(X, \diamond)$ is uniformly supported if all of its elements are supported by the same finite set of atoms.

A subset $U$ of an invariant set $(X, \diamond)$ is finitely supported by a set $T \subseteq A$ if and only if $\pi \star U \subseteq U$ for all $\pi \in \operatorname{Inv}(T)$, i.e., if and only if $\pi \diamond u \in U$ for all $\pi \in \operatorname{Inv}(T)$ and all $u \in U$. This is because any permutation of atoms has a finite order, and so for $\pi \in P_{A}$ we have that there is $m \in \mathbb{N}$ with $\pi^{m}=I d_{A}$, from which we get $\pi \star U \subseteq U \Leftrightarrow \pi \star U=U$.

Clearly, a finite subset of an invariant set is uniformly supported (by the union of the supports of its elements). For uniformly supported sets we have the following property.

Proposition 1 ([19]).

1. Considering a uniformly supported subset $Y$ of an invariant set $(X, \diamond)$, it follows that $Y$ is finitely supported, with $\operatorname{supp}(Y)=\cup\{\operatorname{supp}(y) \mid y \in Y\}$.

2. Considering a finite subset $Y$ of an invariant set $(X, \diamond)$, it follows that $Y$ is finitely supported, with $\operatorname{supp}(Y)=\cup\{\operatorname{supp}(y) \mid y \in Y\}$.

Definition 3. Let $X$ and $Y$ be invariant sets.

1. A function $f: X \rightarrow Y$ is finitely supported whenever $f \in \wp_{f s}(X \times Y)$.

2. Let us consider a finitely supported subset $U$ of $X$, and a finitely supported subset $V$ of $Y$. Then a function $f: U \rightarrow V$ is finitely supported whenever $f \in \wp_{f s}(X \times Y)$; the set of all finitely supported functions from $U$ to $V$ is denoted by $V_{f_{s}}^{U}$.

3. A binary relation between $X$ and $Y$ is finitely supported if it is finitely supported as an element of the $P_{A}$-set $\wp(X \times Y)$. 
Proposition $2([5])$. Let $(X, \diamond)$ and $(Y, \triangleright)$ be two invariant sets.

1. The set $Y^{X}$ of all functions from $X$ to $Y$ is a $P_{A}$-set with the $P_{A}$-action $\widetilde{\star}: P_{A} \times Y^{X} \rightarrow Y^{X}$ defined by $(\pi \widetilde{\star} f)(x)=\pi \triangleright\left(f\left(\pi^{-1} \diamond x\right)\right)$ for all $\pi \in P_{A}, f \in Y^{X}$ and $x \in X$. A function $f: X \rightarrow Y$ is finitely supported (as in Definition 3) if and only if it is finitely supported with respect to the permutation action $\widetilde{\star}$.

2. Let $U$ be a finitely supported subset of $X$ and $V$ a finitely supported subset of $Y$. A function $f: U \rightarrow V$ is supported by a finite set $T \subseteq A$ if and only if $\pi \diamond x \in U, \pi \triangleright f(x) \in V$ and $f(\pi \diamond x)=\pi \triangleright f(x)$ for all $x \in U$ and all $\pi \in \operatorname{Inv}(T)$.

The notion of the cardinality of a finitely supported set was introduced in [8].

Definition 4. Two finitely supported sets $X$ and $Y$ are equipollent if there is a finitely supported bijective function $f: X \rightarrow Y$.

Theorem 1 ([8]). The equipollence relation is an equivariant equivalence relation over the family of all finitely supported sets.

Definition 5. The cardinality of $X$ (denoted by $|X|)$ is the equivalence class of all finitely supported sets equipollent to $X$.

For two finitely supported sets $X$ and $Y$, we have $|X|=|Y|$ if and only if there exists a finitely supported bijection $f: X \rightarrow Y$. We can define a relation $\leq$ over the family of cardinalities defined by $|X| \leq|Y|$ if and only if there exists a finitely supported one-toone (injective) function from $X$ to $Y$. According to Theorem 2 in [8], it follows that $\leq$ is well-defined and equivariant; it is reflexive, anti-symmetric and transitive, but it is not total. Similarly, the relation $\leq^{\star}$ defined by $|X| \leq^{\star}|Y|$ if and only if there exists a finitely supported onto (surjective) function from $Y$ to $X$ is well-defined and equivariant; it is reflexive and transitive, but it is not anti-symmetric, nor total.

Operations with cardinalities of finitely supported sets were defined in [8].

Definition 6. Let $X$ and $Y$ be two finitely supported subsets of invariant sets. The following operations between cardinalities are defined:

$|X|+|Y|=|X+Y| ; \quad|X| \cdot|Y|=|X \times Y| ;$

$|Y|^{|X|}=\left|Y_{f_{S}}^{X}\right|=\mid\{f: X \rightarrow Y \mid f$ is finitely supported $\} \mid$.

As we proved in [8], these operations are well-defined, namely do not depend on the representatives for the involved equivalence classes modulo the equipollence relation.

The translation of a result from ZF to the framework of finitely supported sets can be realized by involving the ' $T$-finite supports principle' presented in [5] and claiming that 'for any finite subset $T$ of $A$, any structure that can be defined in higher-order logic from $T$-supported structures by involving only $T$-supported constructions is also supported by $T^{\prime}$. The involvement of the related $T$-finite support principle actually implies a step-by-step construction of the support of a structure by using, at every step, the supports of the substructures of a related structure that was constructed in the previous steps. Since cardinalities are well-defined for finitely supported structures (as equivalence classes of an equivariant equivalence relation) and basic operations with cardinalities can be presented in the higher-order logic, we get the following property presented as Proposition 3. A detailed proof can be found in Proposition 9 of [8].

Proposition 3. If $X, Y$ and $Z$ are finitely supported subsets of invariant sets, then we have the following properties:

1. $|X|^{|Y| \cdot|Z|}=\left(|X|^{|Y|}\right)^{|Z|}$;

2. $|X|^{|Y|+|Z|}=|X|^{|Y|} \cdot|X|^{|Z|}$;

3. $(|Y| \cdot|Z|)^{|X|}=|Y|^{|X|} \cdot|Z|^{|X|}$. 
We extend a result of [5] from invariant sets to finitely supported sets.

Theorem 2. If $(X, \diamond)$ is a finitely supported subset of an invariant set $(Z, \diamond)$, then there is an injective (one-to-one) function from $\wp_{f s}(X)$ onto $\{0,1\}_{f_{s^{\prime}}}^{X}$ finitely supported by supp $(X)$.

The proof is a rather easy exercise of using the $T$-finite support principle.

\section{Binary Relations between Atomic Sets}

This section presents the main results of the paper. Primarily, we prove some counting properties for finitely supported relations between infinite atomic sets. We use the following result presented in [19].

Theorem 3. Let us consider two finitely supported subsets $X$ and $Y$ of an invariant set Z. If neither $X$ nor $Y$ contain uniformly supported infinite subsets, then $X \times Y$ does not contain $a$ uniformly supported infinite subset.

Lemma 1. Let us consider a finite subset $T=\left\{t_{1}, \ldots, t_{n}\right\}$ of an invariant set $(U, \diamond)$, and a finitely supported subset $X$ of an invariant set $(V, \triangleright)$. If $X$ does not include a uniformly supported infinite subset, then the function space $X_{f_{S}}^{T}$ does not have a uniformly supported infinite subset.

Proof. We show that there is a finitely supported injection $g$ from $X_{f_{s}}^{T}$ into $X^{|T|}$. For $f \in X_{f^{\prime}}^{T}$, it is defined $g(f)=\left(f\left(t_{1}\right), \ldots, f\left(t_{n}\right)\right)$. Obviously, $g$ is injective (it is also surjective). Let $\pi \in \operatorname{Inv}\left(\operatorname{supp}\left(t_{1}\right) \cup \ldots \cup \operatorname{supp}\left(t_{n}\right) \cup \operatorname{supp}(X)\right)$. Due to the fact that $\pi$ is bijective, then $\pi^{-1} \in \operatorname{Inv}\left(\operatorname{supp}\left(t_{1}\right) \cup \ldots \cup \operatorname{supp}\left(t_{n}\right) \cup \operatorname{supp}(X)\right)$. Thus, $g(\pi \widetilde{\star} f)=\left(\pi \triangleright f\left(\pi^{-1} \diamond\right.\right.$ $\left.\left.t_{1}\right), \ldots, \pi \triangleright f\left(\pi^{-1} \diamond t_{n}\right)\right)=\left(\pi \triangleright f\left(t_{1}\right), \ldots, \pi \triangleright f\left(t_{n}\right)\right)=\pi \otimes g(f)$ for all $f \in X_{f_{\mathcal{S}^{\prime}}}^{T}$, where $\otimes$ is the $P_{A}$-action on $X^{|T|}$. Hence $g$ is finitely supported. Using the relation supp $\left(x_{1}\right) \cup$ $\ldots \cup \operatorname{supp}\left(x_{n}\right)=\operatorname{supp}\left(\left(x_{1}, \ldots, x_{n}\right)\right)$ for all $x_{1}, \ldots, x_{n} \in X$, we have that $X^{|T|}$ (meaning the $|T|$-times Cartesian product of $X)$ does not include a uniformly supported infinite subset. Contrarily, $X$ should include itself a uniformly supported infinite subset (which contradicts the hypothesis).

Theorem 4. Let us consider a finitely supported subset $X$ of an invariant set $(Y, \diamond)$ such that $X$ does not include a uniformly supported infinite subset and let $T \in \wp_{\text {fin }}(A)$. Then there exist at most finitely many T-supported functions from $A$ to $X$.

Proof. By contradiction, we assume that for the finite set $T \subseteq A$ there are infinitely many functions $f: A \rightarrow X$ that are supported by $T$.

We have that each $T$-supported function $f: A \rightarrow X$ may be uniquely decomposed into a pair of two T-supported functions $\left.f\right|_{T}$ and $\left.f\right|_{A \backslash T}$ that are the restrictions of $f$ to $T$ and $A \backslash T$, respectively. This comes from Proposition 2, because both $T$ and $A \backslash T$ are supported by $T$, and so for an arbitrary $\sigma \in \operatorname{Inv}(T)$, as $f$ is supported by $T$, we have $\sigma(a) \in T,\left.f\right|_{T}(\sigma(a))=f(\sigma(a))=\sigma \diamond f(a)=\left.\sigma \diamond f\right|_{T}(a), \forall a \in T$, and $\sigma(b) \in A \backslash T$, $\left.f\right|_{A \backslash T}(\sigma(b))=f(\sigma(b))=\sigma \diamond f(b)=\left.\sigma \diamond f\right|_{A \backslash T}(b), \forall b \in A \backslash T$.

According to Lemma 1 , there are only finitely many functions from $T$ to $X$ supported by $T$. Thus, we should have an infinite family $\mathcal{H}$ of functions $g:(A \backslash T) \rightarrow X$ supported by $T$ (functions $g$ are restrictions of functions $f$ to $A \backslash T$ ). Choosing an element $x \in$ $A \backslash T$ and considering an arbitrary $T$-supported function $g:(A \backslash T) \rightarrow X$, for each $\pi \in \operatorname{Inv}(T \cup\{x\})$ we get $\pi \diamond g(x)=g(\pi(x))=g(x)$ (according to Proposition 2). This means that $g(x)$ is supported by $T \cup\{x\}$. However, in $X$ there exist at most finitely many elements supported by $T \cup\{x\}$. Thus, there is $n \in \mathbb{N}$ such that $h_{1}(x), \ldots, h_{n}(x)$ are distinct in $X$. Let us consider $h \in \mathcal{H}$ with $h(x) \in\left\{h_{1}(x), \ldots, h_{n}(x)\right\}$ for $h_{1}, \ldots, h_{n} \in \mathcal{H}$, and an arbitrary $y \in A \backslash T$ (meaning that the transposition $(x y)$ fixes $T$ pointwise). Then, there is $i \in\{1, \ldots, n\}$ such that $h(x)=h_{i}(x)$. Given that $h$ and $h_{i}$ are supported by $T$, and $(x y) \in$ $\operatorname{Inv}(T)$, we get $h(y)=h((x y)(x))=(x y) \diamond h(x)=(x y) \diamond h_{i}(x)=h_{i}((x y)(x))=h_{i}(y)$. 
This finally leads to $h=h_{i}$, because $y$ was arbitrarily chosen from their domain of definition. Therefore, we get a finite $\mathcal{H}=\left\{h_{1}, \ldots, h_{n}\right\}$ which is a contradiction.

Corollary 1. Let us consider a finitely supported subset $X$ of an invariant set $(Y, \diamond)$ such that $X$ does not contain a uniformly supported infinite subset and let $T \in \wp_{\text {fin }}(A)$. Then there exist at most finitely many $T$-supported functions from $A^{n}$ to $X$ where $n \in \mathbb{N}$ and $A^{n}$ is the n-times Cartesian product of $A$.

Proof. We must prove that the set $X_{f_{s}}^{A^{n}}$ of finitely supported functions from $A^{n}$ to $X$ does not have a uniformly supported infinite subset, whenever $n \in \mathbb{N}$. The proof is by induction on $n$. For $n=1$, the claim is true according to Theorem 4. Let us assume that $X_{f_{s}}^{A^{k-1}}$ does not include a uniformly supported infinite subset for some $k \in \mathbb{N}, k \geq 2$. According to Proposition 3, we have $\left|X_{f s}^{A^{k}}\right|=\left|X_{f_{s}}^{A^{k-1} \times A}\right|=|X|^{\left|A^{k-1} \times A\right|}=|X|^{\left|A^{k-1}\right| \cdot|A|}=\left(|X|^{\left|A^{k-1}\right|}\right)^{|A|}=$ $\left|\left(X_{f s}^{A^{k-1}}\right)_{f s}^{A}\right|$. Thus, there is a finitely supported bijection between $X_{f_{s}}^{A^{k}}$ and $\left(X_{f_{s}}^{A^{k-1}}\right)_{f s}^{A}$. However, according to Theorem $4,\left(X_{f_{s}}^{A^{k-1}}\right)_{f_{s}}^{A}$ does not include a uniformly supported infinite subset, because the set $T=X_{f_{s}}^{A^{k-1}}$ does not include a uniformly supported infinite subset (according to the inductive hypothesis).

Corollary 2. Let $T \in \wp_{\text {fin }}(A)$. There exist at most finitely many $T$-supported subsets of $A^{n}$, whenever $n \in \mathbb{N}$.

Proof. We should prove that there are at most finitely many $T$-supported elements of the power set $\wp_{f s}\left(A^{n}\right)$, i.e., $\wp_{f s}\left(A^{n}\right)$ does not have a uniformly supported infinite subset, whenever $n \in \mathbb{N}$. According to Theorem 2, we have $\left|\wp_{f s}\left(A^{n}\right)\right|=\left|\{0,1\}_{f s}^{A^{n}}\right|$. The result is obtained from the previous Corollary, because $\{0,1\}$ is finite and it cannot include a uniformly supported infinite subset.

Now we present the main results of the paper.

Theorem 5. Given an arbitrary finite set $S$ of atoms, there exist at most finitely many $S$-supported relations between $\wp_{n}(A)$ and $\wp_{m}(A)$, where $m, n \in \mathbb{N}^{\star}$ and $\wp_{k}(A)$ denotes the family of all $k$-sized subsets of $A$.

Proof. A relation between $\wp_{n}(A)$ and $\wp_{m}(A)$ is a subset of $\wp_{n}(A) \times \wp_{m}(A)$. Therefore, such a relation $R$ is of form $R=\{(X, Y) \mid X \in \mathcal{F}, Y \in \mathcal{G}\}$ with $\mathcal{F} \subseteq \wp_{n}(A)$ and $\mathcal{G} \subseteq \wp_{m}(A)$. Clearly, if $S$ is the empty set, the only equivariant relation between $\wp_{n}(A)$ and $\wp_{m}(A)$ is $\left\{(X, Y) \mid X \in \wp_{n}(A), Y \in \wp_{m}(A)\right\}$. Let us assume that $|S| \geq 1$ (the claims are also valid for $S=\varnothing)$. The first term in a pair from a relation $R$ is $X=\left\{a_{1}, \ldots, a_{i}, b_{i+1}, \ldots, b_{n}\right\}$ with $a_{1}, \ldots, a_{i} \in S$ and $b_{i+1}, \ldots, b_{n} \in A \backslash S$, and the second term in a pair from a relation $R$ is $Y=\left\{c_{1}, \ldots, c_{j}, d_{j+1}, \ldots, d_{m}\right\}$ with $c_{1}, \ldots, c_{j} \in S$ and $d_{j+1}, \ldots, d_{m} \in A \backslash S$. For any $b \in A \backslash S, b \neq b_{i+1}, \ldots, b_{n}$, we have that $\left(b b_{i+1}\right) \in \operatorname{Inv}(S)$ and, since $S$ supports $R$, we get that $\left\{a_{1}, \ldots, a_{i}, b, b_{i+2}, \ldots, b_{n}\right\}=\left(b b_{i+1}\right) \star X$ should also be the first term in a pair from $R$. By repeatedly applying this procedure, for any different atoms $x_{i+1}, \ldots, x_{n} \in A \backslash S$, we get that $\left\{a_{1}, \ldots, a_{i}, x_{i+1}, \ldots, x_{n}\right\}$ should also be the first term in a pair from $R$. More formally, let us consider the arbitrary different atoms $x_{i+1}, \ldots, x_{n} \in A \backslash S$. We may reorder the finitely many elements $b_{i+1}, \ldots, b_{n}$ and $x_{i+1}, \ldots, x_{n}$ (choice is not involved since we order finite families) such that either $\left\{b_{p} \mid p \in\{i+1, \ldots, n\}\right\} \cap\left\{x_{p} \mid p \in\{i+1, \ldots, n\}\right\}=\varnothing$, or we get an index $k \in\{i+1, \ldots, n\}$ having the properties that $b_{p}=x_{p}$ for all $p \in\{i+1, \ldots, k\}$ and $\left\{b_{p} \mid p \in\{k+1, \ldots, n\}\right\} \cap\left\{x_{p} \mid p \in\{k+1, \ldots, n\}\right\}=\varnothing$. We define a (finite) permutation $\pi$ of $A$ by taking $\pi\left(b_{p}\right)=x_{p}$ for all $p \in\{i+1, \ldots, n\}, \pi(a)=a$ for $a \in A \backslash\left\{b_{i+1}, \ldots, b_{n}\right\}$ if the index $k$ does not exist, or, respectively, by taking $\pi\left(b_{p}\right)=x_{p}$ for all $p \in\{k+1, \ldots, n\}$, $\pi(a)=a$ for $a \in A \backslash\left\{b_{k+1}, \ldots, b_{n}\right\}$ if the index $k$ exists. Clearly, $\pi \in \operatorname{Inv}(S)$ and $\pi$ is finitely supported since it interchanges only finitely many elements from $A$. Since $S$ supports $R$, 
we get $\left\{a_{1}, \ldots, a_{i}, x_{i+1}, \ldots, x_{m}\right\}=\pi \star X$ should be the first term in a pair from $R$. Thus, the choice of $X$ is completely determined by the choice of $a_{1}, \ldots, a_{i} \in S$. Similarly, the choice of $Y$ is completely determined by the choice of $c_{1}, \ldots, c_{j} \in S$. The number $P$ of possibilities to choose the pair $(X, Y)$ is:

$$
\begin{aligned}
& \left(C_{|S|}^{0}+C_{|S|}^{1}+\ldots+C_{|S|}^{n}\right) \cdot\left(C_{|S|}^{0}+C_{|S|}^{1}+\ldots+C_{|S|}^{m}\right) \text { if } n, m \leq|S| \\
& \left(C_{|S|}^{0}+C_{|S|}^{1}+\ldots+C_{|S|}^{n}\right) \cdot 2^{|S|} \text { if } n \leq|S| \text { and }|S|<m ; \\
& 2^{|S|} \cdot\left(C_{|S|}^{0}+C_{|S|}^{1}+\ldots+C_{|S|}^{m}\right) \text { if } m \leq|S| \text { and }|S|<n ; \\
& 2^{|S|} \cdot 2^{|S|}=2^{2|S|} \text { if }|S|<m, n
\end{aligned}
$$

where, by definition, $C_{x}^{l}=\frac{x !}{l !(x-l) !}$ for $x \geq l$, and $y !=1 \cdot 2 \cdot \ldots \cdot y$ for $y \geq 1,0$ ! $=1$. Finally, there are at most $2^{P}$ relations between $\wp_{n}(A)$ and $\wp_{m}(A)$ supported by $S$.

Theorem 6. Given an arbitrary finite set $S$ of atoms, there exist at most finitely many $S$-supported relations between $A^{k}$ and $A^{l}$, where $k, l \in \mathbb{N}^{\star}$.

Proof. A relation between $A^{k}$ and $A^{l}$ is a subset of $A^{k} \times A^{l}$. There exists an equivariant bijection between $\wp_{f_{S}}\left(A^{k} \times A^{l}\right)$ and $\wp_{f_{s}}\left(A^{k+l}\right)$. According to Corollary 2 , since $\wp_{f_{\mathcal{S}}}\left(A^{k+l}\right)$ does not include a uniformly supported infinite subset, it results that $\wp_{f_{s}}\left(A^{k} \times A^{l}\right)$ does not include a uniformly supported infinite subset. Therefore, there are at most finitely many elements from $\wp_{f s}\left(A^{k} \times A^{l}\right)$ (i.e., at most finitely many subsets of $\left.A^{k} \times A^{l}\right)$ that are supported by $S$.

Theorem 7. Given an arbitrary finite set $S$ of atoms, there exist at most finitely many $S$-supported relations between $T_{n}(A)$ and $T_{m}(A)$, where $m, n \in \mathbb{N}^{\star}$ and $T_{k}(A)$ denotes the family of all $k$-sized injective tuples of $A$.

Proof. This result is a direct consequence of Theorem 6 because there exists the equivariant identity injection between $T_{k}(A)$ and $A^{k}$ whenever $k \in \mathbb{N}^{\star}$. However, it could also involve a similar approach as in Theorem 5 to provide the precise number of the related $S$-supported relations. More exactly, a relation $R$ between $T_{n}(A)$ and $T_{m}(A)$ is of form $R=\{(X, Y) \mid X \in$ $\mathcal{F}, Y \in \mathcal{G}\}$ with $\mathcal{F} \subseteq T_{n}(A)$ and $\mathcal{G} \subseteq T_{m}(A)$. The only equivariant relation between $T_{n}(A)$ and $T_{m}(A)$ is $\left\{(X, Y) \mid X \in T_{n}(A), Y \in T_{m}(A)\right\}$. Let $X$ be the left term in a pair from an $S$-supported relation $R$. Assume $|S| \geq 1$ (for notation reasoning although the claims are obviously valid for $S=\varnothing$ ), and suppose there are $p$ columns of the injective tuple $X$ that are occupied by certain fixed different elements from $A \backslash S$. By applying appropriate transpositions, all the injective tuples from $T_{n}(A)$ having the related $p$ columns occupied by arbitrary different elements from $A \backslash S$ should be terms occupying the left position in tuples from $R$. Therefore, the choice of $X$ is completely determined by the choice of the $n-p$ columns occupied by elements of $S$ and by the choice of the elements of $S$ occupying the related $n-p$ columns. We get $2^{\sum_{i=1}^{n} C_{n}^{i} A_{|S|}^{i} \cdot \sum_{j=1}^{m} C_{m}^{j} A_{|S|}^{j}} S$-supported relations, where $A_{n}^{k}=n(n-1) \ldots(n-k+1)$ if $n \geq k$ and $A_{n}^{k}=0$ if $n<k$.

Theorem 8. Let $m, n \in \mathbb{N}^{\star}$. Given an arbitrary finite set $S$ of atoms, there exist at most finitely many $S$-supported relations between $\wp_{n}(A)$ and $A^{m}$, and at most finitely many $S$-supported relations between $A^{n}$ and $\wp_{m}(A)$.

Proof. A relation between $A^{n}$ and $\wp_{m}(A)$ is a subset of the Cartesian product $A^{n} \times$ $\wp_{m}(A)$. We have $\left|\wp_{f s}\left(A^{n} \times \wp_{m}(A)\right)\right|=2^{\left|A^{n} \times \wp_{m}(A)\right|}=2^{\left(\left|A^{n}\right| \cdot\left|\wp_{m}(A)\right|\right)}=\left(2^{\left|\wp_{m}(A)\right|}\right)^{\left|A^{n}\right|}=$ $\left(\left|\wp_{f_{\mathcal{S}}}\left(\wp_{m}(A)\right)\right|\right)^{\left|A^{n}\right|}=\left|\left(\wp_{f_{\mathcal{S}}}\left(\wp_{m}(A)\right)\right)_{f_{S}}^{A^{n}}\right|$. Thus, there is a finitely supported bijection between $\wp_{f_{S}}\left(A^{n} \times \wp_{m}(A)\right)$ and $\left(\wp_{f_{s}}\left(\wp_{m}(A)\right)\right)_{f_{S}}^{A^{n}}$.

We claim that $\wp_{f_{s}}\left(\wp_{m}(A)\right)$ does not include a uniformly supported infinite subset, and so, by Corollary 1 , we have that $\left(\wp_{f_{s}}\left(\wp_{m}(A)\right)\right)_{f_{s}}^{A^{n}}$ does not include a uniformly supported infinite subset. Let $\mathcal{F}$ be a subset of $\wp_{f_{\mathcal{S}}}\left(\wp_{m}(A)\right)$ uniformly supported by a cer- 
tain finite set of atoms $T$. The elements of $\mathcal{F}$ are subsets of $\wp_{m}(A)$. We want to prove that $\mathcal{F}$ is finite, i.e., there are at most finitely many subsets of $\wp_{m}(A)$ supported by $T$. Assume that $|T| \geq 1$, otherwise the problem is trivial (there is only one equivariant subset of $\wp_{m}(A)$, namely $\left.\wp_{m}(A)\right)$. Let $\mathcal{G}$ be a subset of $\wp_{m}(A)$ supported by $T$. Let $X \in \mathcal{G}$, $X=\left\{a_{1}, \ldots, a_{i}, b_{i+1}, \ldots, b_{m}\right\}$ with $a_{1}, \ldots, a_{i} \in T$ and $b_{i+1}, \ldots, b_{m} \in A \backslash T$. Let us consider the arbitrary different atoms $x_{i+1}, \ldots, x_{m} \in A \backslash T$. We may reorder the finitely many elements $b_{i+1}, \ldots, b_{m}$ and $x_{i+1}, \ldots, x_{m}$ (choice is not involved since we order finite families) such that either $\left\{b_{j} \mid j \in\{i+1, \ldots, m\}\right\} \cap\left\{x_{j} \mid j \in\{i+1, \ldots, m\}\right\}=\varnothing$, or we obtain an index $k \in\{i+1, \ldots, m\}$ having the properties that $b_{j}=x_{j}$ for all $j \in\{i+1, \ldots, k\}$ and $\left\{b_{j} \mid j \in\{k+1, \ldots, m\}\right\} \cap\left\{x_{j} \mid j \in\{k+1, \ldots, m\}\right\}=\varnothing$. We define a (finite) permutation $\pi$ of $A$ by taking $\pi\left(b_{j}\right)=x_{j}$ for all $j \in\{i+1, \ldots, m\}, \pi(a)=a$ for $a \in A \backslash\left\{b_{i+1}, \ldots, b_{m}\right\}$ if the index $k$ does not exist, or, respectively, by taking $\pi\left(b_{j}\right)=x_{j}$ for all $j \in\{k+1, \ldots, m\}$, $\pi(a)=a$ for $a \in A \backslash\left\{b_{k+1}, \ldots, b_{m}\right\}$ if the index $k$ exists. Clearly $\pi \in \operatorname{Inv}(T)$ and $\pi$ is finitely supported since it interchanges only finitely many elements from $A$. Since $T$ supports $\mathcal{G}$, we get $\left\{a_{1}, \ldots, a_{i}, x_{i+1}, \ldots, x_{m}\right\}=\pi \star X \in \mathcal{G}$.

Therefore, $\mathcal{G}$ is completely determined by the choice of the elements from $T$ belonging to the members of $\mathcal{G}$. We have therefore at most $2^{\sum_{i=1}^{m} C_{|T|}^{i}}$ (we consider $C_{p}^{l}=0$ if $p<l$ ) ways to define $\mathcal{G}$ such that $\mathcal{G}$ is supported by $T$. Our claim is proved. Thus, we finally obtain that $\wp_{f s}\left(A^{n} \times \wp_{m}(A)\right)$ does not include a uniformly supported infinite subset, and so it contains at most finitely many elements supported by $S$. The second part of this theorem follows in a similar way because $\left.\mid \wp_{f_{s}}\left(\wp_{n}(A) \times A^{m}\right)\right)|=| \wp_{f_{s}}\left(A^{m} \times \wp_{n}(A)\right) \mid$.

Theorem 9. Let $X$ and $Y$ be two finitely supported subsets of an invariant set $Z$ such that neither $X$ nor $Y$ contain a uniformly supported infinite subset. Given an arbitrary finite set $S$ of atoms, there exist at most finitely many S-supported finite relations between $X$ and $Y$.

Proof. A finite relation between $X$ and $Y$ is a finite subset of $X \times Y$, i.e., an element of $\wp_{\text {fin }}(X \times Y)$. Denote $L=X \times Y$. According to Theorem 3, set $L$ does not include a uniformly supported infinite subset. By contradiction, assume that the set $\wp_{\text {fin }}(L)$ includes an infinite subset $\mathcal{F}$ such that all the elements of $\mathcal{F}$ are supported by the same finite set $T$. Thus, $\operatorname{supp}(K) \subseteq T$ for all $K \in \mathcal{F}$. Considering an arbitrary $K \in \mathcal{F}$, we have $\operatorname{supp}(K)=\cup_{x \in K} \operatorname{supp}(x)$ (from Proposition 1 ), and so $K$ has the property $\operatorname{supp}(x) \subseteq T$ for all $x \in K$. Given that $K$ has been arbitrarily chosen from $\mathcal{F}$, it results that every element from each set belonging to $\mathcal{F}$ is supported by $T$, and so $\underset{K \in \mathcal{F}}{\cup} K$ is a uniformly supported subset of $L$ (all elements being supported by $T$ ). Obviously, $\cup_{K \in \mathcal{F}} K$ is infinite since $\mathcal{F}$ is infinite. This is contrary to the hypothesis that $L$ does not include a uniformly supported infinite subset. Finally, we conclude that for a given subset $S$ of atoms there are only finitely many elements of $\wp_{\text {fin }}(L)$ supported by $S$.

Theorem 10. Given an arbitrary, non-empty, finite set $S$ of atoms, there exist at most finitely many $S$-supported functions from $A^{m}$ to $\wp_{\text {fin }}(A)$ (where $m$ is an arbitrary positive integer), but there are infinitely many $S$-supported relations between $S$ and $\wp_{\text {fin }}(A)$.

Proof. The first part results from Corollary 1 , because $\wp_{\text {fin }}(A)$ does not include a uniformly supported infinite subset (for any finite set $S$ of atoms, the finite subsets of $A$ supported by $S$ are precisely the subsets of $S$ ). Now, considering $a \in A$, for any $n \in \mathbb{N}$, the relation $R_{n}=\left\{(a, X) \mid X \in \wp_{n}(A)\right\}$ (with $\wp_{n}(A)$ the family of all $n$-sized subsets of $A$ ) is $\{a\}$ supported, and so it is $S$-supported due to the fact that $a \in S$. This is because $\wp_{n}(A)$ is equivariant for any $n \in \mathbb{N}$ (since permutations of $A$ are bijective, an $n$-sized subset of $A$ is transformed into another $n$-sized subset of $A$ by using a permutation of $A$ ), and so for $\pi \in \operatorname{Inv}(\{a\})$ we have $\pi \otimes(a, X)=(\pi(a), \pi \star X))=(a, \pi \star X)$ with $\pi \star X \in \wp_{n}(A)$, for any $X \in \wp_{n}(A)$. Thus, $\pi \otimes(a, X) \in R_{n}$ for all $(a, X) \in R_{n}$, and so $\pi \star R_{n}=R_{n}$. 
Theorem 11. Given an arbitrary, non-empty, finite set $S$ of atoms, there are at most finitely many $S$-supported functions from $A^{m}$ to $T_{\text {fin }}(A)$ (where $m$ is an arbitrary positive integer), but there exist infinitely many $S$-supported relations between $S$ and $T_{f i n}(A)$, where $T_{\text {fin }}(A)$ is the set of all finite injective tuples of atoms.

Proof. $T_{\text {fin }}(A)$ does not include a uniformly supported infinite subset because the finite injective tuples of atoms supported by a finite set $S$ are only those injective tuples formed by elements of $S$, being at most $1+A_{|S|}^{1}+A_{|S|}^{2}+\ldots+A_{|S|}^{|S|}$ such tuples. The first part of the result now follows from Corollary 1 . Now, let us consider $a \in A$. For any $n \in \mathbb{N}$, the relation $R_{n}=\left\{(a, X) \mid X \in T_{n}(A)\right\}$ with $T_{n}(A)$ the family of all $n$-sized injective tuples of $A$ is $\{a\}$-supported (and so it is $S$-supported since $a \in S$ ). This is because $T_{n}(A)$ is equivariant for any $n \in \mathbb{N}$ (since permutations of $A$ are bijective, an $n$-sized injective tuple of $A$ is transformed into another $n$-sized injective tuple of $A$ by using a permutation of $A$ ), and so for $\pi \in \operatorname{Inv}(\{a\})$ we have $\pi \otimes(a, X)=(\pi(a), \pi \star X))=(a, \pi \star X)$ with $\pi \star X \in T_{n}(A)$, for any $X \in T_{n}(A)$. Thus, $\pi \otimes(a, X) \in R_{n}$ for all $(a, X) \in R_{n}$, and so $\pi \star R_{n}=R_{n}$.

Theorem 12. Given an arbitrary, non-empty, finite set $S$ of atoms, there are at most finitely many $S$-supported functions from $A^{m}$ to $\wp_{f_{s}}(A)$ (where $m$ is an arbitrary positive integer), but there exist infinitely many $S$-supported relations between $S$ and $\wp_{f s}(A)$.

Proof. $\wp_{f_{s}}(A)$ does not include a uniformly supported infinite subset because the elements of $\wp_{f_{S}}(A)$ supported by a finite set $S$ are precisely the subsets of $S$ and the supersets of $A \backslash S$. The first part of the result follows from Corollary 1, and the second part follows from Theorem 10.

\section{Conclusions}

In this article we work in the framework of finitely supported structures and present some counting properties for finitely supported binary relations between infinite atomic sets. Firstly, we presented some finiteness properties of sets which seem to be very large. We proved that function spaces $\wp_{f i n}(A)_{f_{s}}^{A^{m}}, T_{f i n}(A)_{f_{S}}^{A^{m}}, \wp_{f_{s}}(A)_{f_{s}}^{A^{m}}$ (where $m$ is an arbitrary positive integer) do not contain uniformly supported infinite subsets. More generally, $Z^{A^{m}}$ does not contain a uniformly supported infinite subset for each $m \in \mathbb{N}$, whenever $Z$ is a finitely supported set that does not contain a uniformly supported infinite subset. Then we proved that the set of all finitely supported relations between $A^{k}$ and $A^{l}$, the set of all finitely supported relations between $\wp_{k}(A)$ and $\wp_{l}(A)$, and the set of all finitely supported relations between $\wp_{k}(A)$ and $A^{l}$ (for $k, l$ positive integers) do not contain uniformly supported infinite subsets.

Uniformly supported sets are of interest because they involve boundedness properties of supports, meaning that the support of each element in a uniformly supported set is contained in the same finite set of atoms; in this way, all the individuals in an infinite uniformly supported family can be characterized by involving only the same finitely many characteristics. If any uniformly supported subset of a set is finite, the related set can be easier handled in a computable manner [6]. In fact, the sets that do not contain uniformly supported infinite subsets (i.e., the sets mentioned above) are Dedekind-finite in the framework of finitely supported structures, i.e., they do not contain finitely supported countably infinite subsets. Therefore, these sets satisfy the properties of Dedekind-finite sets presented in [8,20] (e.g., Theorem 6 in [8]). Thus, we proved that $\wp_{f_{s}}(X \times Y)$ and $Z^{A^{m}}$ are Dedekind-finite whenever $X$ and $Y$ are chosen from the sets $A^{k}, \wp_{m}(A), \wp_{n}(A)$ and $A^{l}$, $k, l . m, n \in \mathbb{N}$, and $Z$ is a finitely supported Dedekind-finite set.

Finally, we proved that the sets of finitely supported relations between $A$ and $\wp_{\text {fin }}(A)$, as well as between $A$ and $T_{\text {fin }}(A)$ are both Dedekind-infinite in the framework of finitely supported structures, namely they contain uniformly supported infinite subsets. 
Author Contributions: The authors (A.A. and G.C.) contributed equally to this work. All authors have read and agreed to the published version of the manuscript.

Funding: This research received no external funding.

Conflicts of Interest: The authors declare that there is no conflict of interest.

\section{References}

1. Barwise, J. (Ed.) Handbook of Mathematical Logic; North-Holland: Amsterdam, The Netherlands, 1977.

2. Jech, T.J. The Axiom of Choice; Studies in Logic and the Foundations of Mathematics; North-Holland: Amsterdam, The Netherlands, 1973.

3. Fraenkel, A. Zu den Grundlagen der Cantor-Zermeloschen Mengenlehre. Math. Ann. 1922, 86, 230-237. [CrossRef]

4. Lindenbaum, A.; Mostowski, A. Über die Unäbhangigkeit des Auswahlsaxioms und Einiger seiner Folgerungen. Comptes Rendus Séances Société Sci. Lettres Vars. 1938, 31, 27-32.

5. Alexandru, A.; Ciobanu, G. Finitely Supported Mathematics: An Introduction; Springer: Cham, Switzerland, 2016.

6. Alexandru, A.; Ciobanu, G. Foundations of Finitely Supported Structures: A Set Theoretical Viewpoint; Springer: Cham, Switzerland, 2020.

7. Pitts, A.M. Nominal Sets Names and Symmetry in Computer Science; Cambridge University Press: Cambridge, UK, 2013.

8. Alexandru, A.; Ciobanu, G. Various forms of infinity for finitely supported structures. Arch. Math. Log. 2021. [CrossRef]

9. Gabbay, M.J. A general mathematics of names. Inf. Comput. 2007, 205, 982-1011. [CrossRef]

10. Howard, P.; Rubin, J.E. Consequences of the Axiom of Choice; Mathematical Surveys and Monographs; American Mathematical Society: Providence, RI, USA, 1998; Volume 59.

11. Barwise, J. Admissible Sets and Structures: An Approach to Definability Theory; Perspectives in Mathematical Logic; Springer: Cham, Switzerland, 1975; Volume 7.

12. Abramsky, S.; Ghica, D.R.; Murawski, A.S.; Ong, L.C.H.; Stark, I.D.B. Nominal games and full abstraction for the $v$-calculus. In Proceedings of the 19th IEEE Symposium on Logic in Computer Science, Turku, Finland, 17 July 2004; pp. 150-159.

13. Petrisan, D. Investigations into Algebra and Topology over Nominal Sets. Ph.D. Thesis, University of Leicester, Leicester, UK, 2011.

14. Urban, C. Nominal techniques in Isabelle/HOL. J. Autom. Reason. 2008, 40, 327-356. [CrossRef]

15. Bengston, J.; Parrow, J. Formalising the $\pi$-calculus using nominal logic. Log. Methods Comput. Sci. 2009, 5, 1-36.

16. Bojanczyk, M.; Klin, B.; Lasota, S.; Torunczyk, S. Turing machines with atoms. In Proceedings of the 28th Symposium on Logic in Computer Science, New Orleans, LA, USA, 25-28 June 2013; pp. 183-192.

17. Tarski, A. What are logical notions? Hist. Philos. Log. 1986, 7, 143-154. [CrossRef]

18. Horne, R.; Tiu, A.; Aman, B.; Ciobanu, G. De Morgan Dual Nominal Quantifiers Modelling Private Names in Non-Commutative Logic. ACM Trans. Comput. Log. 2019, 20, 22. [CrossRef]

19. Alexandru, A.; Ciobanu, G. Uniformly supported sets and fixed points properties. Carpathian J. Math. 2020, 36, 351-364. [CrossRef]

20. Alexandru, A.; Ciobanu, G. Properties of the atoms in finitely supported structures. Arch. Math. Log. 2020, 59, 229-256. [CrossRef] 